Agric. Biol. Chem., 42 (3), 515 521, 1978

\title{
Chemical and Pharmacological Properties of Vasopressor Phospholipid in Crude Soybean Lecithin
}

\author{
Akira Tokumura, Yasue Akamatsu, Sadaji Yamada \\ and Hiroaki TsukatanI \\ Faculty of Pharmaceutical Sciences, Tokushima University, \\ Shomachi, Tokushima, Japan
}

Received July 6, 1977

\begin{abstract}
Commercial soybean lecithin has a potent hypertensive effect on anesthetized rats. Crude soybean lecithin was separated into two fractions with respect to solubility in cold acetone. The acetone-insoluble fraction containing most pressor activity was separated into two further active fractions by column chromatography on silicic acid. The active fraction eluted with chloroform-methanol mixture (4:6) was further purified by gel filtration on Sephadex LH-20. The purified pressor substance had phosphorus and was labile to alkali. Its chromatographic and partition behaviors indicate that it may be a polar acidic phospholipid. The shortlasting pressor response produced by the active lipid was not reduced by mechanical injury to the central nervous system or treatment with an $\alpha$-adrenergic blocking agent or a ganglionblocking agent.
\end{abstract}

Commercial soybean lecithin, a mixture of phospholipids containing a number of neutral lipids and glycolipids, has been utilized in food chemistry because of its emulsifying properties, antioxidant effect and its nutritional value. It has also been known that soybean lecithin contains an unknown depressor substance. McQuarrie and Anderson ${ }^{1)}$ obtained a depressor-free preparation with emulsifying property using an anion-exchange resin. This depressor substance seems to be a lipid-like substance, although it has remained unidentified.

Nutritional and pharmacological interests led us to investigate the vasoactive substance in commercial soybean lecithin, which resulted in the observation that soybean lecithin had a potent hypertensive effect on rats. The present paper describes some chemical and pharmacological properties of this pressor substance.

\section{METHODS}

Biological assay. For general assay, male Wistar rats weighing $200 \sim 250 \mathrm{~g}$ were used. Under urethane anesthesia $(2 \mathrm{~g} / \mathrm{kg}$, i. p.), the left jugular vein was cannulated with a polyethylene tube for injection of materials from a microsyringe. The trachea and right carotid artery were also cannulated, and the latter was connected to a direct-writing mercury manometer. The arterial pressure response was recorded on a slowmoving fumed drum. Materials to be tested were suspended in $0.05 \mathrm{ml}$ of Ringer solution. When necessary, $2 \%$ Tween 80 was added to this solution.

For preparation of spinal rats, the following technique $^{2)}$ was employed. Rats, weighing $380 \sim 400 \mathrm{~g}$, were anesthetized with urethane $(1.8 \mathrm{~g} / \mathrm{kg}, \mathrm{i} . \mathrm{p}$.). A spinal section was cut at $\mathrm{C}-2$, and the brain was destroyed. The rats were artificially ventilated with a respiration pump. Blood pressure was recorded from the carotid artery via a cannula connected to a mercury manometer. Test samples were injected into one femoral vein.

Solvent fractionation. Commercial soybean lecithin $(10 \mathrm{~g})$ was dissolved into $100 \mathrm{ml}$ of chloroform. After addition of five volumes of acetone, the mixture was centrifuged at $2000 \mathrm{rpm}$ for $25 \mathrm{~min}$ and the precipitate was washed three times with $100 \mathrm{ml}$ of cold acetone. The combined supernatant was concentrated under reduced pressure to dryness. The washed precipitate was redissolved with $50 \mathrm{ml}$ of chloroform. The solution was mixed with five volumes of cold ethanol under shaking. Centrifugation separated the acetoneinsoluble materials into two fractions, "Lecithin" fraction and "Cephalin" fraction.

Purification of pressor substance. The acetoneinsoluble materials were fractionated into some classes of phospholipid on silicic acid (Mallinckrodt, AR 100 
mesh). Silicic acid was activated at $110^{\circ} \mathrm{C}$ overnight and poured into a column $(50 \mathrm{~cm} \times 2 \mathrm{~cm})$ as a suspension in chloroform. Discontinuous gradient elution was carried out with chloroform-methanol mixtures of increasing polarity as follows: 1 chloroform, 2 chloroform-methanol $(19: 1, v / v), 3$ chloroformmethanol $(9: 1), 4$ chloroform-methanol $(8: 2), 5$ chloroform-methanol $(7: 3), 6$ chloroform-methanol (6:4), 7 chloroform-methanol $(4: 6), 8$ chloroformmethanol $(2: 8)$ and 9 methanol. Fractions of $100 \mathrm{ml}$ were collected. The fraction eluted with solvent 3 contained one-third of total activity. The remains of pressor activity were recovered in fraction eluted with solvent 7 , and were further purified by chromatography on Sephadex LH-20 (Phamacia Fine Chemicals) with chloroform-methanol mixture $(1 ; 1)$ as a developing solvent system. Fractions of $10 \mathrm{ml}$ were collected.

The lipids in the chromatographed fractions were monitored by thin-layer chromatography on silica gel (Merck, silica gel plate 60, $0.25 \mathrm{~mm}$ thick) with the following acidic, basic and neutral solvent systems: chloroform-methanol-acetic acid-water $(60: 35: 1: 8)$, chloroform-methanol-28\% ammonia $(65: 35: 5)$ and chloroform-methanol-water $(65: 25: 4)$. Exposure of the plate in iodine vapor or charring with ethanolic sulfuric acid detected lipids. For detection of phospholipids, the modified molybdenum blue method described by Vaskovsky and Svetashev ${ }^{3)}$ was employed. The following specific spray reagents were also used: Dragendorff reagent for choline, benzidine reagent for amide, ninhydrin reagent for amino group and periodate-Schiff reagent for vicinal hydroxyl-containing compounds.

For further purification of the pressor substance on thin-layer chromatogram, the materials separated by Sephadex LH-20 chromatography were spotted on a preparative plate (Merck, silica gel plate $60,2 \mathrm{~mm}$ thick). The desired parts were removed horizontally from the plate and the compounds were extracted with methanol. The methanol suspension was poured into a stuall column $(50 \mathrm{~mm} \times 10 \mathrm{~mm})$ packed with small amount of Hyflo super-cel. The methanolic eluate $(50 \sim 100 \mathrm{ml})$ was filtered on a glass-filter to remove fine particles of silica gel, and the filtrate was evaporated to dryness. The residue was injected intravenously into rats.
Partition behavior of pressor substance. The highly purified pressor substance was dissolved in organic solvents. To the respective organic solutions equal volumes of water at various $\mathrm{pHs}$ were added. After shaking for $5 \mathrm{~min}$ at room temperature, the partitioned organic phases were separated and evaporated to dryness under reduced pressure. An aliquot of each residue was dissolved in Ringer solution and injected intravenously into the rat.

Analytical procedures. Phosphorus was determined by the procedure described by Chalvardjian and Rudnicki. ${ }^{4)}$ Total nitrogen was measured by the semimicro Kjeldahl method. The free amino group in unhydrolyzed phospholipids was determined by the ninhydrin method. ${ }^{5)}$ Choline was examined by the molybdenum blue method reported by Wheelden and Collins, ${ }^{B)}$ Glycerol content was determined as described by Renkonen. ${ }^{7)}$ Lipid ester was estimated according to the method of Snyder and Stephens. ${ }^{87}$

\section{RESULTS}

The commercial soybean lecithin used in the present experiments proved to be contaminated with large amounts of simple lipids and complex lipids other than phosphatidyl choline. After removal of most of the simple lipids by solvent fractionation with acetone, the complex lipids (acetone-insoluble fraction) were separated into two classes, "Cephalin" fraction and "Lecithin" fraction with respect to solubility in cold ethanol. Pressor activity was predominantly recovered in the latter fraction as shown in Table I.

The complex lipid fraction(acetone-insoluble fraction) was chromatographed on a column of silicic acid with the chloroform-methanol mixtures, as shown in Fig. 1. Fraction 2 consisted of some phospholipids stained with periodate-Schiff spray reagent. The eluate of the chloroform-methanol mixture $(9: 1)$ con-

Table I. Solvent Fractionation of Commercial Soybean Lecithin

\begin{tabular}{|c|c|c|c|c|c|c|}
\hline Fraction & $\begin{array}{l}\text { Weight } \\
\text { (mg) }\end{array}$ & $\begin{array}{l}\text { Total-P } \\
\text { (umole) }\end{array}$ & $\begin{array}{l}\text { Total-N } \\
\text { ( } \mu \text { mole) }\end{array}$ & $\begin{array}{c}\text { Total- } \\
\text { amino-N } \\
(\mu \text { mole })\end{array}$ & $\begin{array}{l}\text { Total- } \\
\text { choline } \\
(\%)\end{array}$ & $\begin{array}{c}\text { Pressora) }^{a)} \\
\text { activity }\end{array}$ \\
\hline Crude preparation & 1548 & 910 & 910 & 300 & 100.0 & \\
\hline Acetone-soluble fraction & 681 & 48 & 89 & 23 & 7.2 & - \\
\hline Ethanol-soluble fraction & 661 & 715 & 647 & 230 & 89.2 & 150 \\
\hline Ethanol-insoluble fraction & 171 & 155 & 62 & 29 & 3.5 & - \\
\hline
\end{tabular}

a) One unit of pressor activity corresponds to pressor activity produced by $0.4 \mu \mathrm{g} / \mathrm{kg}$ of noradrenalin. 


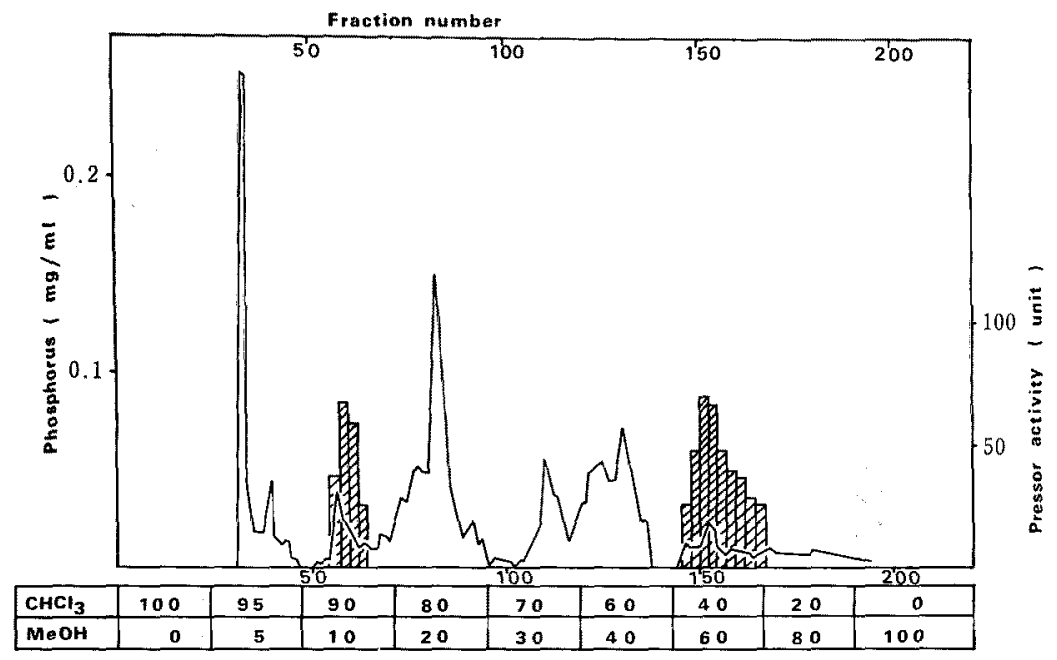

Fig. 1. Silicic Acid Column Chromatography of Complex Lipid Fraction in Soybean Lecithin. 漛, pressor activity; -, phosphorus content.

tained approximately one-third of the total activity. With increased polarity of eluting solvent, several common phospholipids were eluted successively as follow: phosphatidyl ethanolamine (Fraction 4), phosphatidyl inositol (Fraction 5) and phosphatidyl choline (Fraction 6). The remaining pressor activity was recovered from Fraction 7 by elution with a chloroform-methanol mixture $(4: 6)$.

Fraction 7 was further purified by gel filtration on a Sephadex LH-20 column with a chloroform-methanol mixture $(1: 1)$. In the chromatogram shown in Fig. 2, the major phosphorus peak appeared in tube numbers 19 22, containing primarily phosphatidyl choline and lysophosphatidyl ethanolamine. Most pressor activity was distributed broadly in tube numbers $35 \sim 45$ together with a second minor phosphorus peak. A concentration of about 700-fold of active substance was obtained by solvent fractionation and two column chromatographies.

The active fraction was located on a silica gel plate with chloroform-methanol-water (65:35:5) as a developing solvent system. There are two major phospholipids and one minor sugar-like substance (Fig. 3). The activity migrated to zone 5 . A spot positive to molybdenum blue reagent in zone 5 was not stained with Dragendorff, benzidine, periodate-

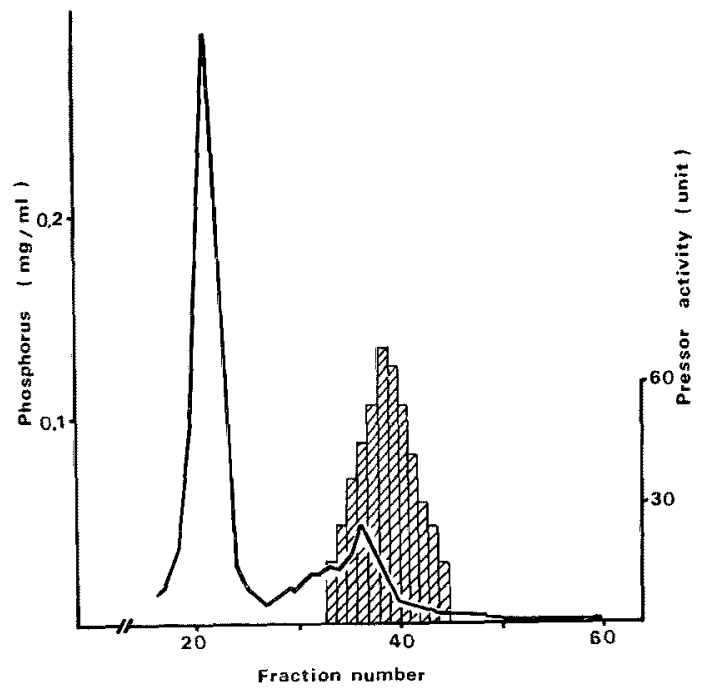

Fig. 2. Purification of Active Phospholipid by Sephadex LH-20 Column Chromatography.

应, pressor activity; -, phosphorus content.

Schiff, $\alpha$-naphtol or ninhydrin spray reagent. The phosphorus/glycerol/ester molar ratio of the active fraction proved to be $1.00: 1.04: 0.99$ in chemical analyses.

Figure 4 shows the effect of $\mathrm{pH}$ on partition properties of the partially-purified pressor substance between the organic and aqueous phases. Petroleum ether did not extract a significant amount of active substance from 
aqueous phase at any tested hydrogen ion concentration. Its partition into more polar

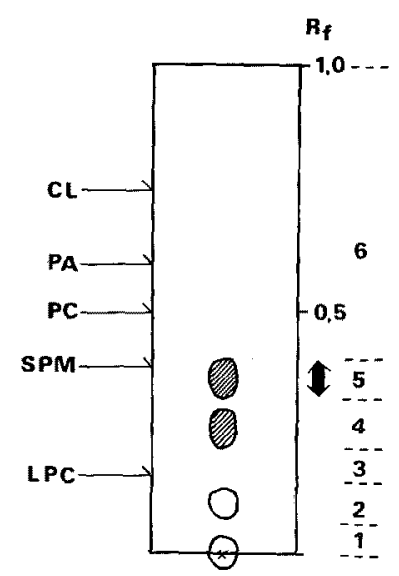

FIG. 3. TLC of Purified Active Fraction on Silica Gel Plate.

Zone 5 contains most of the pressor activity. CL, cardiolipin (from beef heart, Sigma); PA, phosphatidic acid (from egg yolk, Sigma); SPM, sphingomyelin (from bovine brain, Sigma); PC, D, L- $\alpha$-lecithin (dipalmitoyl-, Sigma); LPC, lysolecithin (from egg yolk, Sigma). organic phase was increased by progressive reduction in $\mathrm{pH}$. With increased polarity in organic phases, the recovery of pressor activity increased at all hydrogen ion concentrations. With Folch's solvent, similar tendency was observed. The recovery of activity in the chloroform-rich phase was highly dependent on the $\mathrm{pH}$ of the water-rich phase. These results indicate that the pressor substance is a polar acidic lipid. Furthermore, the pressor substance is more labile to alkali than acid (Fig. 5). On the other hand, no reduction in pressor activity was observed by treatment at $\mathrm{pH} 7$ for $24 \mathrm{hr}$.

The dose-response relationship of pressor substance on an anesthetized rat is shown in Fig. 6 . The maximal blood pressure elicited by this substance was less than that induced by noradrenalin. The effective dose range for producing a progressive increase was also less than that of noradrenalin.

The short-lasting pressor response by the active substance was not characterized by

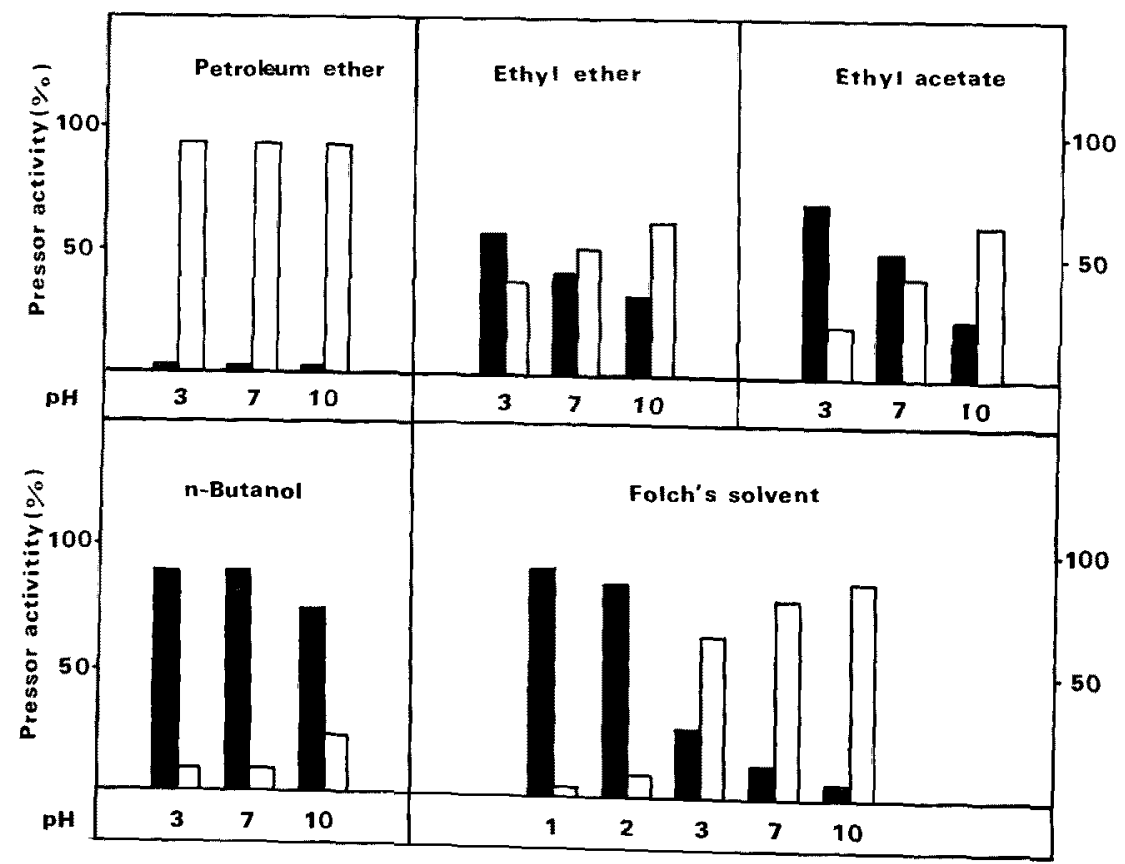

Fig. 4. Effect of pH on the Partition of Purified Pressor Substance between Various Organic and Aqueous Phases.

The blank bar indicates pressor activity in aqueous phase, and the dark bar indicates pressor activity in organic phase. Folch's solvent was prepared by adding 0.2 volume of distilled water to the mixture of chloroform-methanol (2:1). Values are means of duplicate experiments. 


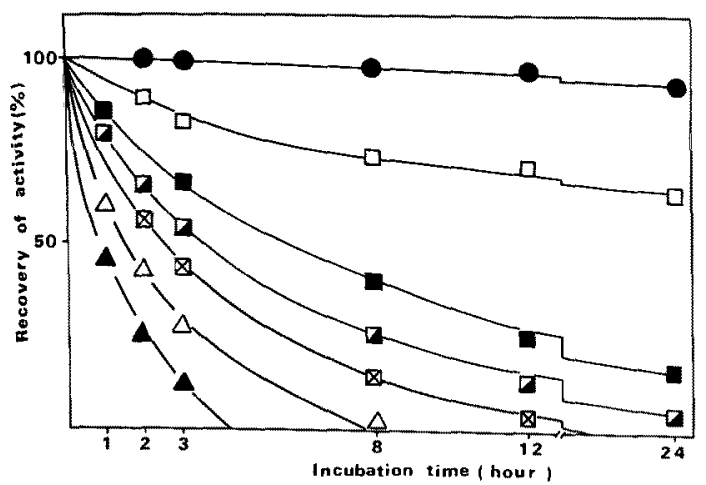

Fig. 5. Stability of Pressor Substance in Distilled Water at Different pHs.

Purified pressor substance (ca. 50 units, see Table I), was dissolved in $5 \mathrm{ml}$ of distilled water at different pHs. The solution was incubated at $37^{\circ} \mathrm{C}$ for $24 \mathrm{hr}$. At each incubation time, aliquots of solution were withdrawn and evaporated to dryness. The residue was dissolved in $0.2 \mathrm{ml}$ of Ringer solution and injected intravenously into rats.

$\mathrm{N}=2, \Delta=\mathrm{pH} \mathrm{1,} \mathbf{\square}=\mathrm{pH} \mathrm{2, \square}=\mathrm{pH} 3, \bullet=\mathrm{pH} 7$, $\mathrm{pH} 11, \otimes=\mathrm{pH} 12, \Delta=\mathrm{pH} 13$.
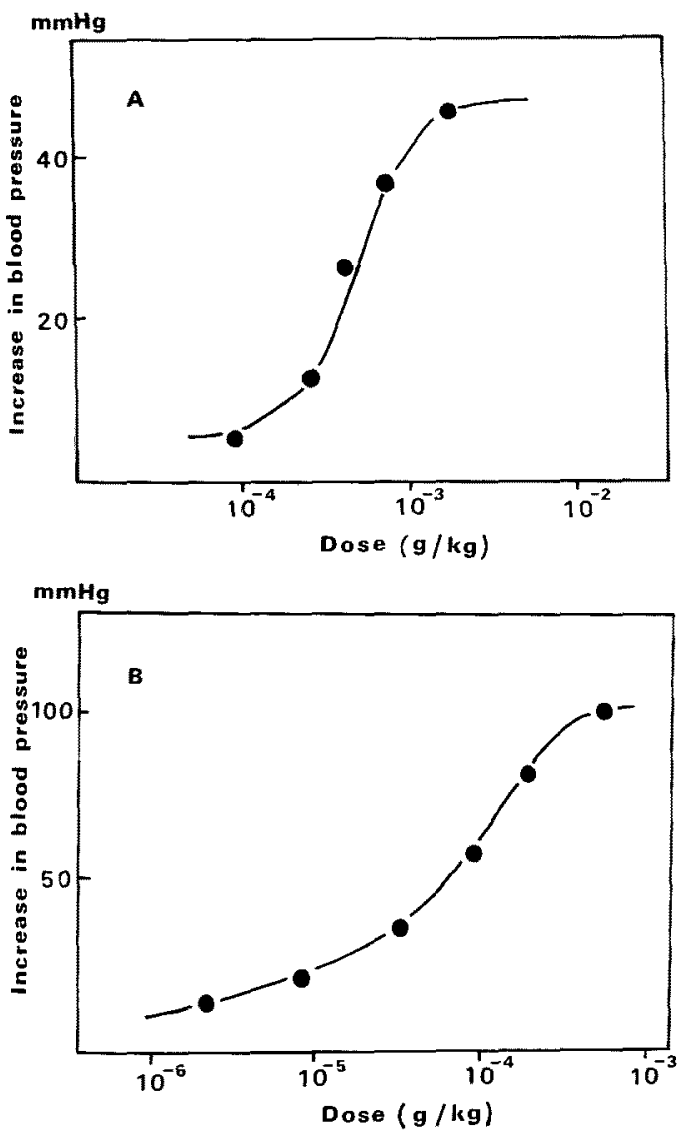

FIG. 6. Dose-response Curves for Pressor Phospholipid (A) and Noradrenalin (B).

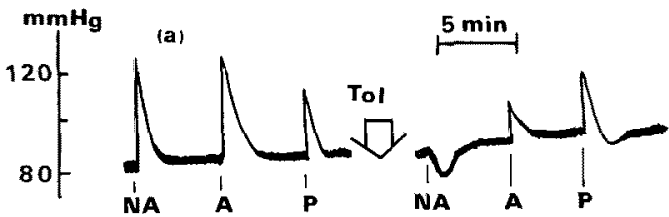

(b)
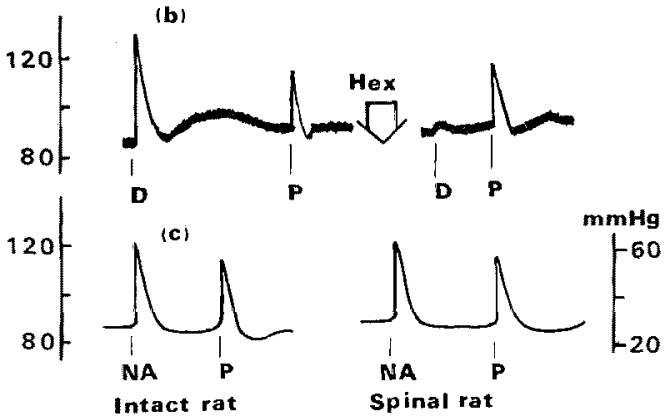

FIG. 7. Effect of Treatment with $\alpha$-Adrenergic Blocking Agent (a) or with Ganglion-blocking Agent (b) and Mechanical Injury of the Central Nervous System (C).

Antagonism by each blocker was confirmed by injection of noradrenalin, adrenalin and 1,1-dimethyl-4phenylpiperazinium, and then vasoactive phospholipid was administered into rat. $\mathrm{P}$, pressor phospholipid $(200 \mu \mathrm{g} / \mathrm{kg})$; NA, noradrenalin $(0.4 \mu \mathrm{g} / \mathrm{kg})$; A, adrenalin $(0.4 \mu \mathrm{g} / \mathrm{kg}) \mathrm{D}, 1,1$-dimethyl-4-phenylpiperazinium $(0.1 \mathrm{mg} / \mathrm{kg})$; Hex, hexamethonium (5 mg/kg, i. p.); Tol, tolazoline $(5 \mathrm{mg} / \mathrm{kg}$, i. p.). Arrow indicates time of injection.

tachyphylaxis and was not blocked by a ganglion-blocking agent(hexamethonium) or an $\alpha$-adrenergic blocking agent(tolazoline). Mechanical injury of the central nervous system caused no detectable response reduction (Fig.7).

\section{DISCUSSION}

Potent vasoactive lipids have not been found in vegetable sources to the best of our knowledge. Some acute vasoactive analogs of fatty acids having hypotensive effect(prostaglandins $^{9 \sim 11}$ and slow reacting substance- $\mathrm{C}^{121}$ ) were obtained from animal sources. Among them, F-type prostaglandins are unique compounds which are hypertensive to rats and dogs and hypotensive to cats and rabbits. The pressor substance found in commercial soybean lecithin seems to be different from F-type prostaglandins on the basis of chemical pro- 
perties, such as poor solubility in cold acetone and chromatographic patterns.

In investigating unknown pharmacologically active lipids, Vogt ${ }^{13}$ stated that it was necessary to use the partition procedure between organic and aqueous phase to exclude deceptive pharmacological effects due to contamination of potent active non-lipid materials. Although the pressor substance in soybean lecithin was highly purified, the possibility still remains that a certain lipid is contaminated with some known pressor substance, such as noradrenalin in the highly purified lipid preparation. ${ }^{14}$ This possibility is not excluded, but the pressor substance in soybean lecithin was apparently distinguishable from sympathetic amines, because the pressor response of rats to pressor lipid was mediated by neither efferent nor afferent sympathetic nervous system. The partition behavior of the active substance was characteristic of acidic lipids. In Folch's solvent, its recovery in chloroform-rich phase was highly dependent on $\mathrm{pH}$ in aqueous phase, whereas $n$-butanol extracted it quantitatively at neutral and acidic $\mathrm{pH}$. This behavior is unique and similar to that of acidic 1ysophospholipids reported by Bjerve et $a l^{15}{ }^{15}$

The chromatographic behavior of the active substance on Sephadex LH-20 also may reflect its acidic property. Eichberg and Burnham ${ }^{16}$ ? observed that acidic phospholipid moved more slowly on Sephadex LH-20 than neutral phospholipids in chloroform-methanol mixture (2:1). Kisselev $\left.{ }^{17}\right)$ also suggested that Sephadex LH-20 was a weak anion-exchanger in chloroform-methanol mixtures.

Few reports have been published on the occurrence of vasoactive phospholipids in biological sources. Hickler et $\left.a l_{.}{ }^{18}\right)$ showed the existence of a certain pressor substance in acetone-insoluble fraction during identification of prostaglandins from rabbit kidney. This materials could be easily distinguished from prostaglandins, but was not further characterized. Khairallah and Page ${ }^{19}$ also suggested that a pressor lipid from incubated dog plasma was lysophosphatidyl choline, possibly produced from phosphatidyl choline by the ac- tivated plasma phospholipase A. They also observed that synthetic stearoyllysophosphatidyl choline could lower the blood pressure of rats. The inversion in biological activity was explained as due to differences in the fatty acid moiety in the molecules. It is unlikely that our pressor phospholipid is lysolecithin from chromatographic and partition behaviors. Analytical data on the phosphorus-glycerolester ratio of the active phospholipid suggested that it would be a polar acidic phospholipid, probably lysophospholipid, distinguished from other known vasoactive lipids.

The present observation suggests that the pressor response produced by our phospholipid may be mediated by its direct effects on the cardiovascular system, not by activation on the central nervous system. In addition, it would exert effects to sites different from aadrenergic receptor in the peripheral cardiovascular system.

The active phospholipid was highly purified and it produced a significant increase in blood pressure at a dose of $50 \mu \mathrm{g} / \mathrm{kg}$, but further investigation is necessary to determine chemical structure of this interesting vasoactive phospholipid.

Acknowledgment. The authors wish to thank Dr. Hideo Fukuda for his excellent technical assistance in the experiments with spinal rats.

\section{REFERENCES}

1) E. B. McQuarrie and H.P. Anderson, U. S. Patent, 2931818 (1960).

2) H. Kumagai, T. Yui, K. Ogawa aud and H. Oga, Seitai no Kagaku, 5, 132 (1953).

3) V. E. Vaskovsky and V. I. Svetashev, J. Chromatogr., 65, 451 (1972).

4) A. Chalvardjian and E. Rudnicki, Anal. Biochem., 36, 225 (1970).

5) C. H. Lea and D. N. Rhodes, Biochim. Biophys. Acta, 17, 416 (1955).

6) L. W. Wheelden and F. D. Collins, Biochem. J., 70, 43 (1958).

7) O. Renkonen, Biochim. Biophys. Acta, 56, 367 (1962).

8) F. Snyder and N. Stephens, ibid., 34, 244 (1959).

9) D. W. Ducharme, J. R. Weeks and R G. Montgomery, J. Pharmacol. Exp. Ther., 160, 1 (1968).

10) J. Nakano and B. Cole, Am. J. Physiol., 217, 222 
(1969).

11) M. C. Koss, J. W. Gray, M. Davidson and J. Nakano, Eur. J. Pharmacol., 24, 151 (1973).

12) B. B. Vargaftig, E. P. De Miranda and B. Lacoume, Nature (London), 222, 883 (1969).

13) W. Vogt, Pharmacol. Rev., 10, 407 (1958).

14) J. D. Robinson, E. A. Carlini and J. P. Green, Biochem. Pharmacol., 12, 1219 (1963).

15) K. S. Bjerve, L. N. W. Daae and J. Bremer, Anal.
Biochem., 58, 238 (1974).

16) J. Eichberg and J. P. Burnham, J. Lipid Res., 11, $386(1970)$.

17) G. V. Kisseley, Biokhimiya, 34, 483 (1969).

$18)$ R. B. Hickler, D. P. Lauler, C. A. Saravis, A. I. Vagnucci, G. Steiner and G. W. Thorn, Can. Med. Ass. J., 90, 280 (1964).

19) P. A. Khairallah and I. H. Page, Am. J. Physiol., 199, 341 (1960). 